\title{
Numerical investigation of characteristic frequency excited highly underexpanded jets
}

\author{
Xiaopeng $\mathrm{Li}^{\mathrm{a}}{ }^{\mathrm{a}}$, E. Fan ${ }^{\mathrm{a}}$, Wei Yao ${ }^{\mathrm{a}, \mathrm{c}}$, Xuejun Fan ${ }^{\mathrm{a}, \mathrm{c}, *}$ \\ a State Key Laboratory of High Temperature Gas Dynamics, Institute of Mechanics, Chinese Academy of Sciences, Beijing 100190, China \\ ${ }^{\mathrm{b}}$ Research Center of Heat and Mass Transfer, Institute of Engineering Thermophysics, Chinese Academy of Sciences, Beijing 100190, China \\ c School of Engineering Science, University of Chinese Academy of Science, Beijing 100049, China
}

\section{A R T I C L E I N F O}

\section{Article history:}

Received 24 October 2016

Received in revised form 29 December 2016

Accepted 12 January 2017

Available online 17 January 2017

\section{Keywords:}

Fuel jets

Underexpanded jets

Jet mixing control

Excited jets

Large eddy simulation

\begin{abstract}
A B S T R A C T
A highly underexpanded jet with a nozzle pressure ratio of 5.60 is excited simultaneously by the inherent characteristic frequencies in the steady jet of $14.569 \mathrm{kHz}, 37.086 \mathrm{kHz}$, and $45.695 \mathrm{kHz}$ as well as other two reference frequencies of $1.0 \mathrm{kHz}$ and $40.0 \mathrm{kHz}$. The flow characteristic of the excited jets is revealed by comparing to the steady jet using large eddy simulation technique. For low-frequency excitation $\left(f_{e}=\right.$ $1.0 \mathrm{kHz}$ ), the flow and acoustic fields of the forcing jet are similar with the steady jet. However, when the jet is excited by high frequencies, the acoustic source moves to the nozzle exit, and the jet potential core together with the near-field shocks oscillate periodically at the excitation frequency. The excitation at $f_{e}=1.0 \mathrm{kHz}$ increases the mixing area since $y / D=24$ from the nozzle exit, which is contrary to the effect of other high frequencies that enhances the mixing in the near-field region but decreases the mixing area since $y / D=18$. The peak frequency of the excited jets generally becomes identical to the excitation frequency once being excited, except the $f_{e}=1.0 \mathrm{kHz}$ and $f_{e}=40.0 \mathrm{kHz}$ jets. High-order harmonics of the dominant frequency are observed in the pressure spectrum of jets excited by high frequencies, and the dominant mode turns into the axisymmetric mode from the original helical one accordingly. In particular, forcing the jet with the axisymmetric mode of $f_{e}=14.569 \mathrm{kHz}$ provides the fewest shock cells but the largest amplitude in shock oscillation, the most harmonics in the spectrum, and the largest mixing area within $8 \leq y / D \leq 12$.
\end{abstract}

(c) 2017 Elsevier Masson SAS. All rights reserved.

\section{Introduction}

High-pressure jets have widespread applications, especially in energy and propulsion systems. A few of these include injection of gaseous fuel jets in a supersonic combustor [1-3], discharge of chimney gases into the atmosphere [4,5], and thrust control of rockets and V/STOL aircraft via jets [6,7]. In most of these applications, an overall control of the fluid mechanics of the flow is desirable to improve the quality of the end process. In particular for a scramjet, ultrafast mixing of fuel with oxidizer is required due to the limited time and length available in the combustor. Additional performance factors of a scramjet, such as the combustion efficiency, combustion stability, and flammability limits, are all strongly affected by the mixing process between fuel and air [8-10].

Two fuel injection strategies, i.e. transverse and parallel injections, are usually used in scramjet combustors. Transverse injection has good penetration but is inevitably accompanied by shocks

\footnotetext{
* Corresponding author.

E-mail addresses: lxpyfy@163.com (X. Li), xfan@imech.ac.cn (X. Fan).
}

$[11,12]$, which induce excess total pressure loss. Transverse injection also encounters severe cooling problems since the hightemperature reaction zone is generally close to the wall. On the other hand, parallel injection has lower total pressure losses and provides enhanced thrust, but its penetration into the cross stream is generally poor [13]. Due to inherent drawbacks of the two traditional injection techniques, several passive control techniques have been suggested to enhance fuel-air mixing at high speeds $[8,9,14]$. Cavity-based injections [15], step-based [16] or strut-based [1,17] injections, and swept ramp injectors [18,19] are some of the wellstudied designs. Most of these techniques enhance mixing by producing counter-rotating streamwise vortices. For example, Rogers et al. (1998) [20] found that the vorticity is generated in the flow over a ramp due to the baroclinic torque and higher mixing and combustion efficiencies are consequently reached. A comprehensive summary of different mixing enhancement techniques can be found in the review by Seiner et al. (2001) [8] and Gutmark et al. (1995) [14].

Besides the traditional passive control techniques, active control techniques, such as the forced excitation, can manipulate the jet shear layer characteristics and significantly enhance the jet 
mixing [8]. Jet excitation generally refers to the introduction of disturbances to the flow to excite either the shear layer instability or the jet column instability. The most common form of excitation is pulsing the flow with pressure fluctuations to produce periodic fluctuations in mass flow. Over the last few decades, a significant amount of work has been performed to investigate the impact of the excitation signal including its frequency, amplitude and phase on the structure and mixing of the jet. Note that most of these works focused on low speed jets into incompressible subsonic crossflow. For example, Eroglu and Breidenthal (2001) [21] investigated the structure and mixing of pulsed jets into an incompressible crossflow, which are periodically modulated by a square wave. Their measurements showed up to a $70 \%$ increase in jet penetration and 50\% reduction in flame length of the jet at the optimum pulsing frequency. Narayanan et al. (2003) [22] experimentally studied the effect of forcing of a subsonic jet issuing into a subsonic cross flow, and found that exciting a jet at higher frequencies could improve jet spread in the near field, whereas lower frequency enhanced the entrainment and mixing in the far field. Johari (2006) [23] compiled lots of experiments performed in the gaseous or liquid phase, and developed a map to demarcate the dependence regimes on the stroke frequency of the injected slug for pulsed jets in subsonic crossflows. The effect of sinusoidal excitation on incompressible subsonic transverse jets with different jet-to-crossflow velocity ratio $R$ was studied by Davitian et al. (2010) [24]. Their results suggested that even low-to-moderatelevel sinusoidal forcing can control penetration and spread for the jets with relatively large $R$ values. However, such excitation, even at high amplitude, appeared to have a relatively minimal or no effect on jets with low $R$ values. One may also refer the review of the transverse jets and their control by Karagozian (2010) [25] for further details.

The mixing problem is especially important for high-speed propulsion applications, but previous work on underexpanded excited jets is relatively rare. Murugappan et al. (2005) [26] studied the mixing and penetration of a forced supersonic jet in a supersonic Mach 2.0 cross stream using Rayleigh/Mie scattering, and found that the periodically formed large-scale eddies in the jet/free stream in the excited jets provided high jet penetration into the free stream as compared to the baseline. Kouchi et al. (2010) [27] also investigated the penetration characteristics of the pulsed injection into a supersonic crossflow based on schlieren photograph and RANS simulations, and indicated that the vortex rings generated in the pulsed jets with the pulsation frequency above $10 \mathrm{kHz}$ remarkably increased the jet penetration and promoted the mixing. For parallel injections, Raman and Rice (1989) [28] experimentally studied the effect of simultaneous excitation at the fundamental and subharmonic frequencies on the behavior of a circular jet, and found that the two-frequency excitation is more effective than the single-frequency excitation in mixing enhancement. Samimy et al. (2007, 2010) $[29,30]$ forced an axisymmetric perfectly expanded Mach 1.3 jet using plasma actuators, and studied the acoustic and flow characteristic of the excited jets based on the flow velocity and pressure probed. Note that most of these studies are based on simple measurements, and the information about the instantaneous flow characteristic of jets is limited. It is also noticed that the frequencies applied to force the jet in reported literatures can be classified as in low regime and are generally selected randomly. Therefore, a systematic study on the flow characteristic of the excited supersonic underexpanded jets is necessary.

The present work aims to study the flow characteristic of the highly underexpanded jets excited simultaneously at high frequencies using large eddy simulation (LES). The highly underexpanded jet with a nozzle pressure ratio (NPR) of 5.60, an exit Mach number of 1.0 , and an exit Reynolds number around $10^{5}$ is selected as the baseline jet. The inherent peak frequencies in the steady jet of $14.569 \mathrm{kHz}, 37.086 \mathrm{kHz}$, and $45.695 \mathrm{kHz}$ as well as other two reference frequencies of $1.0 \mathrm{kHz}$ and $40.0 \mathrm{kHz}$ are set as the forcing frequencies to excite the jets. In particular, $f=14.569 \mathrm{kHz}$ is consistent with the symmetric mode of the jet, while $f=37.086$ and $45.695 \mathrm{kHz}$ correspond to the helical instability modes. The LES modeling of high-pressure gas injection issued from a threedimensional nozzle geometry is performed using a compressible OpenFOAM solver, astroFoam, with a high resolution mesh. The effect of external excitation on the flow evolution, instantaneous flow field, shock structures, mixing characteristic, and the instability modes of the highly underexpanded jets is investigated.

\section{Methodology}

\subsection{Numerical methods}

The governing equations employed in the current LES are the filtered Navier-Stokes equations describing the conservation of mass, momentum, energy and species:

$$
\begin{aligned}
& \frac{\partial \bar{\rho}}{\partial t}+\frac{\partial \bar{\rho} \tilde{u}_{i}}{\partial x_{i}}=0 \\
& \frac{\partial \bar{\rho} \tilde{u}_{i}}{\partial t}+\frac{\partial \bar{\rho} \tilde{u}_{i} \tilde{u}_{j}}{\partial x_{j}}=-\frac{\partial \bar{P}}{\partial x_{i}}+\frac{\partial \tilde{\tau}_{i j}}{\partial x_{j}}-\frac{\partial \tau_{i j}^{s g s}}{\partial x_{j}} \\
& \frac{\partial \tilde{\rho} \tilde{h}_{s}}{\partial t}+\frac{\partial \tilde{\rho} \tilde{u}_{j} \tilde{h}_{s}}{\partial x_{j}}=\frac{\partial \bar{p}}{\partial t}+\frac{\partial \tilde{u}_{j} \tilde{\tau}_{i j}}{\partial x_{i}}-\frac{\partial \bar{q}_{i}}{\partial x_{i}}-\frac{\partial H_{i}^{s g s}}{\partial x_{i}} \\
& \frac{\partial \bar{\rho} \tilde{Y}_{k}}{\partial t}+\frac{\partial \bar{\rho} \tilde{u}_{j} \tilde{Y}_{k}}{\partial x_{j}}=\frac{\partial}{\partial x_{j}}\left(\bar{\rho} \tilde{D}_{k m} \frac{\partial \tilde{Y}_{k}}{\partial x_{j}}\right)-\frac{\partial \Phi_{k, j}^{s g s}}{\partial x_{j}}
\end{aligned}
$$

where "-" means cell average, “ $\sim$ " means Favre average, $\rho$ is density, $u_{i}$ is the velocity in $x_{i}$ direction, $P$ is the pressure, $\tau_{i j}$ is the viscous stress tensor, $h_{s}$ is the sensible enthalpy per unit mass, $q_{i}$ is the heat flux vector, $Y_{k}$ is the mass fraction, $D_{k m}$ is the equivalent binary mass diffusivity. The pressure is computed from the equation of state:

$\bar{p}=\bar{\rho} R \tilde{T}$

where $\tilde{T}$ is the temperature, $R$ is the gas constant of the mixture gas.

The thermodynamic and transport properties of individual species, such as the enthalpy per unit mass $h_{k}$ and the specific heat at constant pressure $c_{p k}$, are calculated based on NIST-JANAF thermo-physical and transport database [31]. The dynamic viscosity $\mu_{k}$ is computed by Sutherland's law. All terms with superscript sgs in equations (2)-(4) denote sub-grid quantities. In particular, the SGS energy fluxes term, $H_{i}^{\text {sgs }}=\left(\bar{\rho} \widetilde{E_{t} u_{i}}-\bar{\rho} \tilde{E}_{t} \tilde{u}_{i}\right)+\left(\overline{p u_{i}}-\bar{p} \tilde{u}_{i}\right)$, is modeled based on a linear eddy diffusivity assumption as:

$H_{j}^{s g s}=-\bar{\rho} \frac{\nu_{t}}{\operatorname{Pr}_{t}} \frac{\partial \tilde{H}}{\partial x_{j}}=-\bar{\rho} \frac{\nu_{t}}{\operatorname{Pr}_{t}}\left(\frac{\partial \tilde{h}}{\partial x_{j}}+\tilde{u}_{i} \frac{\partial \tilde{u}_{i}}{\partial x_{j}}+\frac{\partial k^{s g s}}{\partial x_{j}}\right)$

where $v_{t}$ is the eddy viscosity. The SGS species fluxes term, $\Phi_{k, j}^{s g s}=$ $\bar{\rho} \widetilde{u_{j} Y_{k}}-\bar{\rho} \tilde{u}_{j} \tilde{Y}_{k}$, is also modeled using the eddy diffusivity assumption as:

$\Phi_{k, i}^{s g s}=-\bar{\rho} \frac{v_{t}}{S c_{t}} \frac{\partial \tilde{Y}_{k}}{\partial x_{i}}$

Generally, one-equation model has advantages on modeling the transitional flows or flows with large scale unsteadiness compared to the "zero" equation models (e.g. Smagorinsky model). Our previous work [35] also indicated that the one-equation model is recommended if focusing on the acoustic properties of underexpanded jets. Therefore, the SGS stress term, $\tau_{i j}^{\text {sgs }}=\bar{\rho}\left(\widetilde{u_{i} u_{j}}-\tilde{u}_{i} \tilde{u}_{j}\right)$, 
is modeled with the sub-grid scale turbulent kinetic energy oneequation model [32] in the present LES as:

$$
\begin{aligned}
& \frac{\partial \bar{\rho} k^{s g s}}{\partial t}+\frac{\partial \bar{\rho} \tilde{u}_{j} k^{s g s}}{\partial x_{j}} \\
& =\frac{\partial}{\partial x_{j}}\left[\bar{\rho}\left(\frac{v_{t}}{\operatorname{Pr}_{t}}+v\right) \frac{\partial k^{s g s}}{\partial x_{j}}\right]-\tau_{i j}^{s g s} \frac{\partial \tilde{u}_{i}}{\partial x_{j}}-C_{\varepsilon} \frac{\bar{\rho}\left(k^{s g s}\right)^{3 / 2}}{\bar{\Delta}}
\end{aligned}
$$

$v_{t}=C_{v} \bar{\Delta} \sqrt{k^{s g s}}$

where $C_{v}$ and $C_{\varepsilon}$ are model constants.

The above equations are solved using a density-based compressible solver astroFoam, which is developed based on the basic rhoCentralFoam solver [33] distributed with OpenFOAM v2.3.0. The astroFoam solver is well established and validated in our previous LES of underexpanded hydrogen jet [34] and nitrogen jets at different NPR [35]. The method used in previous LES for similar problems has been discussed in [35], thus only the main aspects of the astroFoam solver are briefly described here. In astroFoam, the convection-diffusion equation is solved by semi-discrete KT (Kurganov and Tadmor) scheme [36] for shock capturing and turbulence resolving. NVD (Normalised Variable Diagram) scheme based on the Minmod limiter [37] is applied to reconstruct the primitive values at faces to obtain the second order accuracy. Time integration is carried out by the Crank-Nicholson scheme [38], which is second order accurate in time.

\subsection{Simulation setup}

The fuel injection model used in the present study is the same as that presented in our previous studies [34,35]. The computational domain mainly consists of a box of size $50 \times 100 \times 50 \mathrm{~mm}$ in the $x$-, $y$-, and $z$-directions, respectively. The nitrogen jet (mass fraction $Y_{N 2}=1.0$ ) is injected into the quiescent air (with static pressure $p_{\infty}$ and static temperature $T_{\infty}$ ) from an axisymmetric high-pressure nozzle (with total pressure $p_{0}$ and total temperature $T_{0}$ ) with a contoured round orifice of $2.0 \mathrm{~mm}$ in diameter (D), as seen in Fig. 1(a). The height of the nozzle is $20 \mathrm{~mm}$, and the nozzle exit is located at $y=0$ plane.

As indicated by the previous studies [3,7,11,12,34,35,39-42], the spatial resolution in numerical modeling of supersonic flows needs to be rather high. A grid independence study by using two different meshes, i.e. the coarse and fine meshes, is performed in Ref. [35], and it is found that the fine mesh has advantages on capturing the turbulence characteristics and acoustic properties of the underexpanded jets. Therefore, the fine mesh in Ref. [35] is used in the present LES of excited jets, which is shown in Fig. 1(b). The fine mesh is hexahedral and block-structured, and contains about 27.3 million computational cells altogether. The jet potential core is meshed with high resolution by adding a refined region to cover the jet potential core and the jet shear layers. Coarse cells with a resolution of $1.0 \mathrm{~mm}$ in the far field and $0.5 \mathrm{~mm}$ at outflow boundaries are used to introduce additional dissipation and avoid wave reflections from these boundaries. More details on the mesh resolution and its effect on modeling the underexpanded jets can be found in our previous work [35].

The quiescent air is the mixture of $76.699 \%$ nitrogen and $23.301 \%$ oxygen by mass. Initially the temperature, pressure, density, and velocity of the ambient air are all uniform, i.e. $T_{\infty}=$ $300 \mathrm{~K}, P_{\infty}=101325 \mathrm{~Pa}, \rho_{\infty}=1.17 \mathrm{~kg} / \mathrm{m}^{3}, U_{\infty}=0$. The simulation in [35] for a steady underexpanded nitrogen jet at NPR of 5.60 is set as the base case in the present LES. In particular, the pure nitrogen gas is injected at a stagnation temperature of $360.0 \mathrm{~K}$ and a stagnation pressure $0.57 \mathrm{MPa}$ with a sonic speed at the nozzle exit, where the Reynolds number is about Re $\sim 10^{5}$. Details of flow conditions for the steady jet are summarized in Table 1 .

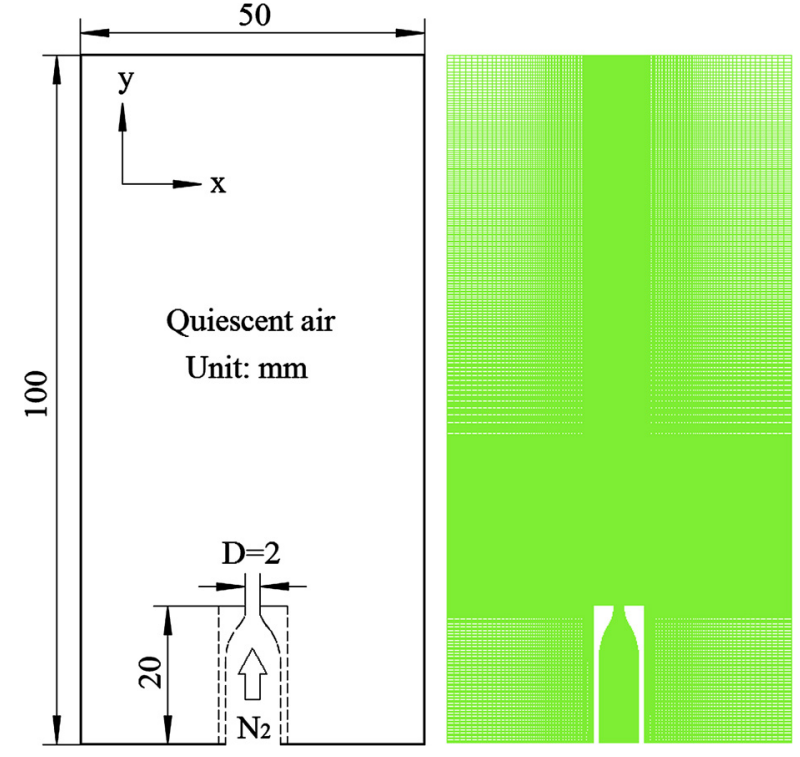

Fig. 1. Computational domain (left) and grid (right) employed for the LES of underexpanded jets.

Table 1

Highly underexpanded jet conditions for the baseline case without excitation.

\begin{tabular}{llll}
\hline Property & Symbol & Value & Unit \\
\hline Mach number at nozzle exit & $M_{1}$ & 1.0 & - \\
Static pressure at nozzle exit & $P_{1}$ & 0.3 & $\mathrm{MPa}$ \\
Stagnation pressure & $P_{0}$ & 0.57 & $\mathrm{MPa}$ \\
Static temperature at nozzle exit & $T_{1}$ & 300.0 & $\mathrm{~K}$ \\
Velocity at nozzle exit & $U_{1}$ & 353.1 & $\mathrm{~m} / \mathrm{s}$ \\
Reynolds number at nozzle exit & $\mathrm{Re}_{1}$ & 1.36 & $\times 10^{5}$ \\
Static pressure ratio & $P_{1} / P_{\infty}$ & 2.96 & - \\
Nozzle pressure ratio (NPR) & $P_{0} / P_{\infty}$ & 5.60 & - \\
\hline
\end{tabular}

In the present study, a sinusoidal disturbance is imposed to the pressure at the nozzle entrance to form the excited jets. The static pressure $P$ at the nozzle entrance varies with time $t$ at exciting frequency $f_{e}$ as:

$P=P_{2}\left[1.0+A_{m} \sin \left(2 \pi f_{e} t\right)\right]$

where $P_{2}$ is the pressure at the nozzle entrance for the steady unexcited jet and takes a value of $0.5674 \mathrm{MPa}, A_{m}$ is the forcing amplitude and is assumed to be 5\% referring to the previous experimental studies [21-28] on subsonic and supersonic excited jet flows, $f_{e}$ is the forcing frequency and will be discussed in detail in the next section.

The computational time step is approximately $\Delta t \approx 1.37 \times$ $10^{-8} \mathrm{~s}\left(\Delta t \cdot a / D=2.42 \times 10^{-3}, a\right.$ is the speed of sound at the nozzle exit), as limited by a maximum Courant-Friedrichs-Lewy (CFL) number of 0.6. This time step is similar with that used by Liu et al. (2009) [39], Dauptain et al. (2010) [40], and Hamzehloo and Aleiferis (2016) [41] in LES of underexpanded jets. The flowthrough time (FTT) for jets washing out the computational domain in streamwise direction is around $0.5 \mathrm{~ms}=200 t_{0}$, with $t_{0}=2.5 \times 10^{-6} \mathrm{~s}=2.5 \mu \mathrm{s}$. The total simulation duration is thus set as $4 \mathrm{FTT}=2.0 \mathrm{~ms}=800 t_{0}$, which is four times the value used by Vuorinen et al. (2013) [42] to ensure long enough quasi-steady period for turbulent statistics. The instantaneous results are saved every $2 t_{0}$, and turbulent statistics are collected for the last three flow-through times (3FTT, 200 $\left.t_{0} \sim 800 t_{0}\right)$. 


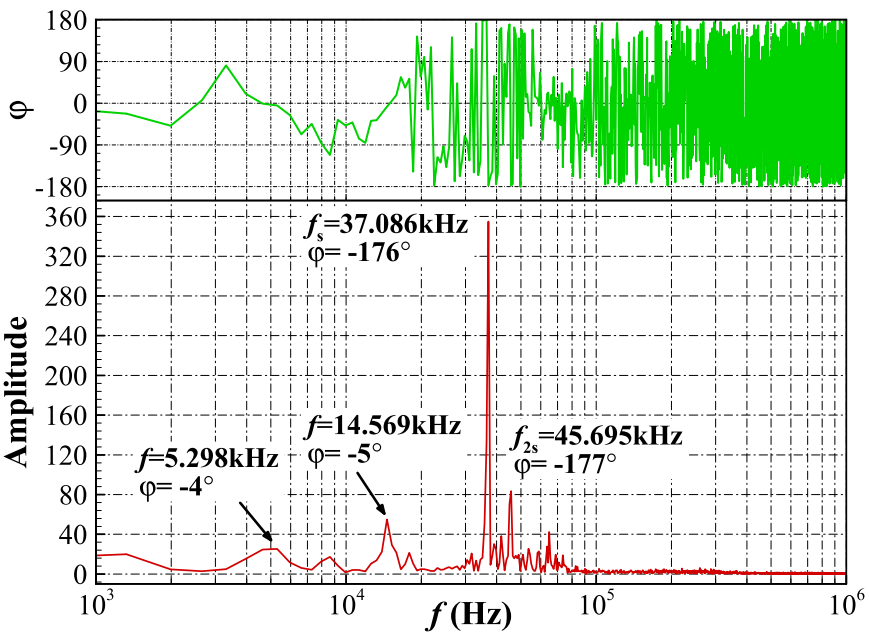

Fig. 2. The cross spectrum and relative phase of pressure fluctuation on either side of the steady underexpanded jet at NPR of 5.60 .

\subsection{Excited frequencies}

An important issue on excited jets is how to select a suitable excitation frequency to obtain better mixing. However, a general consensus on selecting the excitation frequency is not reached although there are numerous experimental studies on subsonic and supersonic excited jet flows so far. Cutler et al. (2001) [43] found experimentally that the effective exciting frequency for jet issued into a supersonic cross flow is between $10 \mathrm{kHz}$ and $50 \mathrm{kHz}$. Cutler et al. (2001) [43] and Kouchi et al. (2010) [27] indicated that the jet flow field excited by too low or high frequencies is similar to the steady jet, which is useless for mixing enhancing. However, the experiment by Randolph et al. (1994) [44] reported a $12 \%$ increase in penetration when forcing a transverse jet in a supersonic cross flow at $1 \mathrm{~Hz}$ compared with the steady jet. Murugappan et al. (2005) [26] chose $900 \mathrm{~Hz}$ and $5 \mathrm{kHz}$ as the excitation frequencies, and found that both of the excited jets show higher entrainment and better mixing characteristics compared to the steady jet without excitation.

Previous studies [45-48] indicated that some characteristic frequencies exist in supersonic underexpanded jets, and Fig. 2 presents the spectrum of pressure fluctuation of the steady underexpanded nitrogen jet for NPR $=5.60$ obtained by our previous LES. As can be seen, the dominant frequency of the steady jet is
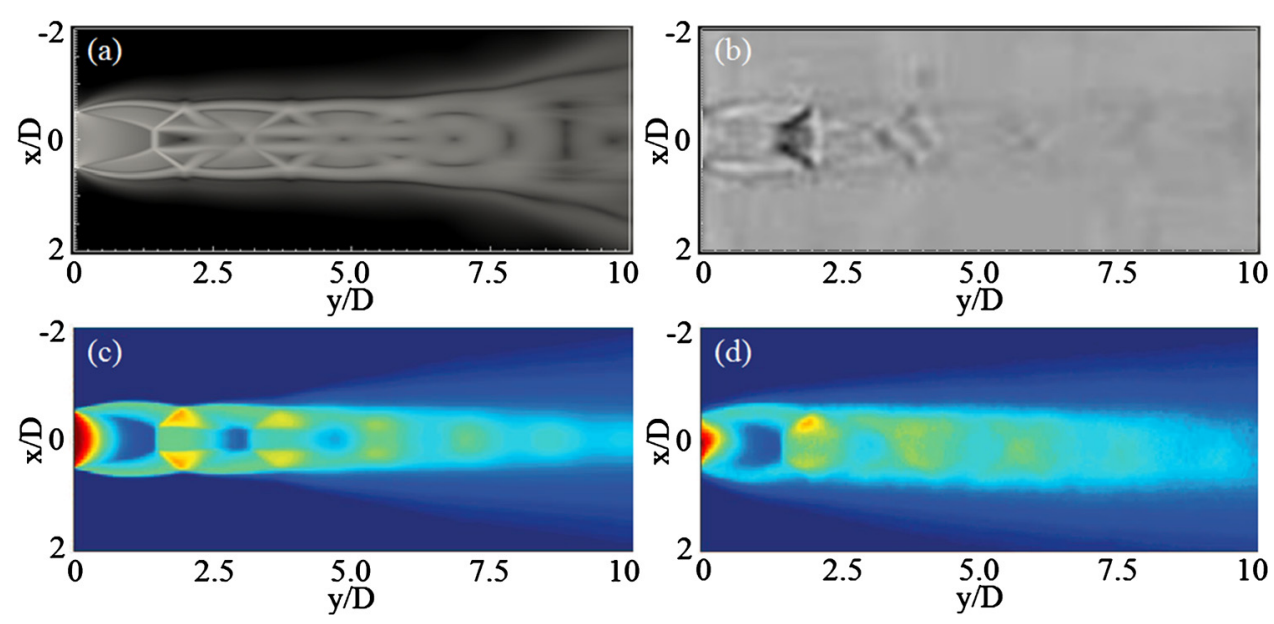

Exciting frequencies $f_{e}(\mathrm{kHz})$ employed to force the jet.

\section{Numerical validation} disk height.

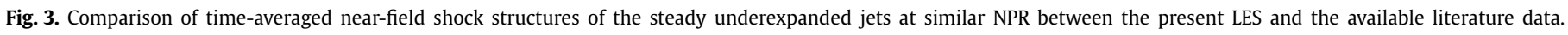

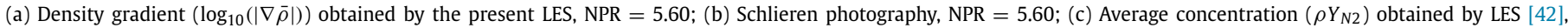
$\mathrm{NPR}=5.5$; (d) Average concentration $\left(\rho Y_{N 2}\right)$ obtained using PLIF (Planar Laser Induced Fluorescence) technique [42], NPR $=5.5$.

\begin{tabular}{llllll}
\hline Case 1 & Case 2 & Case 3 & Case 4 & Case 5 & Case 6 \\
\hline 0 & 1.0 & 14.569 & 37.086 & 40.0 & 45.695 \\
\hline
\end{tabular}

$f_{s}=37.086 \mathrm{kHz}$, which is usually referred to as the shock screech frequency. Another discrete peak of $f_{2 s}=45.695 \mathrm{kHz}$ with amplitude lower than the screech tone is called the secondary shock screech frequency. Both of those two peak frequencies have a phase angle close to $\pm 180^{\circ}$, and correspond to the helical modes. There are also some other discrete frequencies, for example $f=$ $5.298 \mathrm{kHz}$ and $f=14.569 \mathrm{kHz}$ corresponding to the symmetric mode with a phase angle close to $0^{\circ}$. The helical modes are closely related with the jet instability. Thereby, a natural way to select the excitation frequencies is the screech frequencies of those two helical modes. Two other frequencies, $1.0 \mathrm{kHz}$ and $40 \mathrm{kHz}$, are also selected to be the forcing frequencies. In particular, $f_{e}=1.0 \mathrm{kHz}$ is a low exciting frequency, while $f_{e}=40.0 \mathrm{kHz}$ is a high frequency between the shock screech frequency $f_{s}$ and the secondary shock screech frequency $f_{2 s}$. As a summary, Table 2 lists the exciting frequencies used to force the underexpanded jet in the present LES.

The time-averaged near-field shock structures of the steady jet predicted by the current LES is presented in Fig. 3(a). As can be seen, the classical wave structures $[49,50]$ in the near field of a highly underexpanded jet, including the Mach disk, barrel shock, triple point, reflected shock and slip lines, are all well captured by the present LES. Meanwhile, the predicted shock structures are in good agreement with the schlieren photography shown in Fig. 3(b) at the same NPR of 5.60. The present LES result also compares reasonably with the LES modeling and PLIF measurement [42] at a similar NPR of 5.5 shown in Fig. 3(c) and (d) in terms of the Mach

Fig. 4(a) quantitatively compares the time-averaged density along the jet centerline between the LES results for NPR $=5.60$ and the measured data by Panda and Seasholtz (1999) [51] at a similar NPR of 5.74. Note that $\rho_{j}$ is the fully expanded jet density. A good agreement with the experimental data is observed for the first two shocks. The deviation at the third shock may be attributed to the different $\rho_{j} / \rho_{\infty}$ values used in LES and the experiments. On the other hand, the radial profiles of density near the Mach disk predicted by LES are compared with the measurements in Fig. 4(b). 

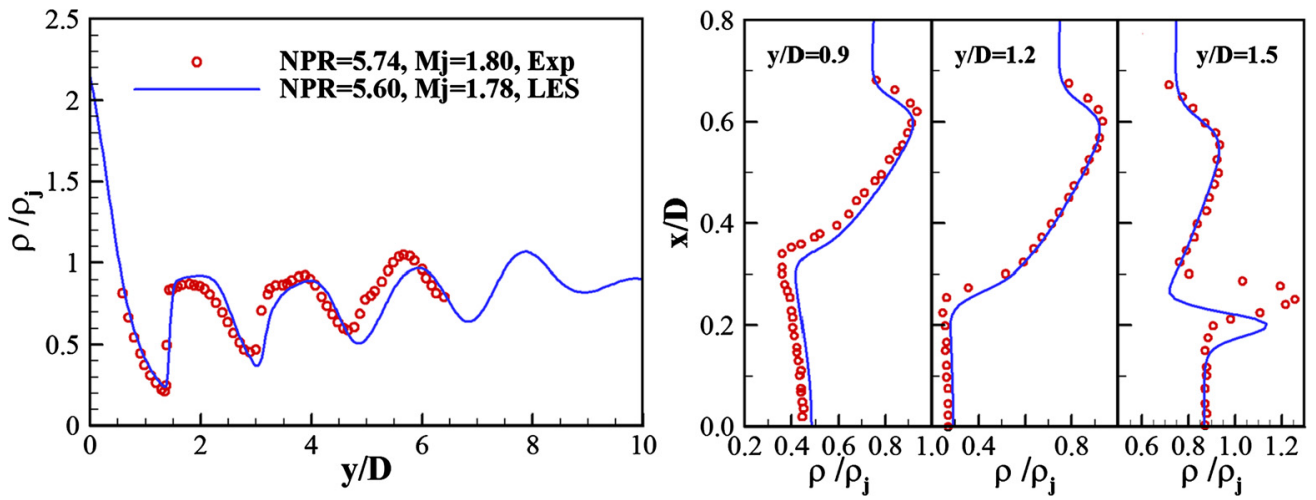

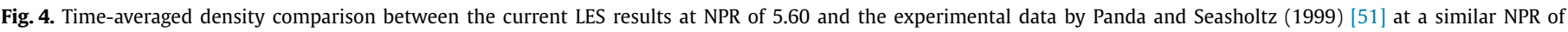
5.74. (a) Left: axial profile, (b) right: radial profiles at different streamwise positions.
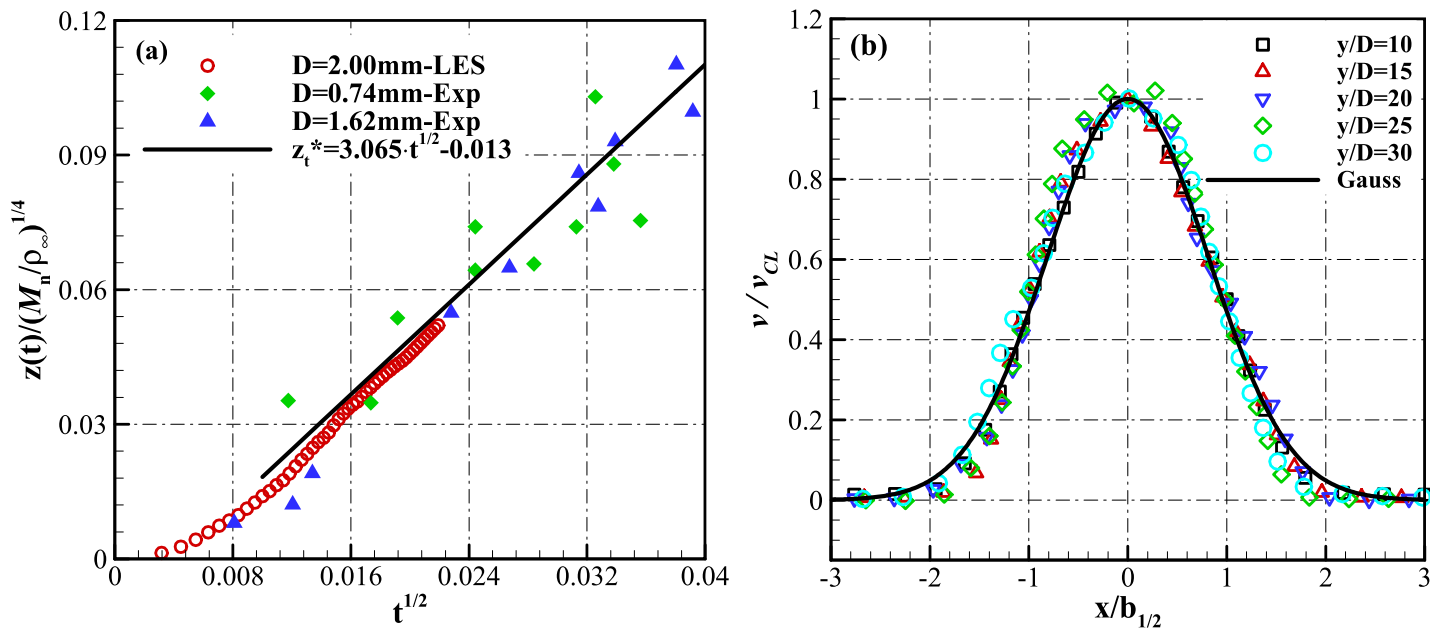

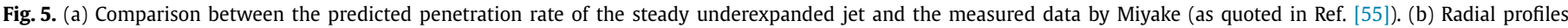
of non-dimensional mean streamwise velocities at different streamwise positions for the steady jet.

The LES results agree well with the measured data at the first two streamwise positions, i.e. $y / D=0.9$ and 1.2 . At $y / D=1.5$, the predicted density peak is at around $x / D=0.2$, which is smaller than the measured value of $x / D=0.25$. This observation suggests that the present LES predicts a narrower Mach disk than the experiment, as also shown in Fig. 3. Similarly, the LES studies performed by Vuorinen et al. (2013) [42] and Hamzehloo and Aleiferis (2016) [52] also reproduced the width of the Mach disk smaller compared to the measurements. The contributing factors, as indicated by Franquet et al. (2015) [53] and explained by Hamzehloo and Aleiferis (2016) [52], can be the differences in nozzle geometry design and simulation setup (e.g. the turbulence levels at the nozzle exit, and the different boundary conditions used) between the LES modeling and the experiments.

Ouellette (1996) [54] investigated experimentally the penetration rates of underexpanded sonic jets at $\operatorname{Re} \sim 5.0 \times 10^{5}$ and found that the penetration of the fuel jets obeys a linear dependency on the square root of time. Fig. 5(a) compares the penetration rate of the steady underexpanded jets between the current LES results and the measurements by Miyake et al. (1983) (as quoted in Ref. [55]). As can be seen, the present LES clearly confirms this linear dependency behavior after the initial turbulent transition. Meanwhile, the penetration rates of underexpanded sonic jets in the near field, which are different with that in the downstream and generally inaccessible experimentally, are also well captured by the current LES modeling.

Previous experimental and numerical studies [56-58] indicated the radial profiles of streamwise velocity are similar for different streamwise positions in the downstream region of the jets, which can be expressed as:

$$
\frac{v}{v_{C L}}=f\left(\frac{x}{b_{1 / 2}}\right)
$$

where $v$ is the streamwise velocity, $v_{C L}$ is the streamwise velocity at the centerline, $x$ is the distance to the centerline, and $b_{1 / 2}$ is the jet half-width. Fig. 5(b) presents the predicted mean streamwise velocity profiles at different streamwise positions for the steady jet, which indicates that this self-similarity property is well resolved by the present LES. More details on the numerical validation and flow analysis of the steady jet can be found in Ref. [35].

\section{Results and discussion}

\subsection{Quasi-steady state jets}

Fig. 6(a) shows the instantaneous contours of density gradient together with the nitrogen mass fraction on the centerline plane for the steady jet, and some of the typical characteristics of underexpanded circular jets are evident. For example, the shocks inside the jet show a wavy up and down oscillation as visualized using schlieren photographs by Panda (1998) [59]. The inner shear layer (the slip lines) persists at about the fourth shock cell, and then merges with the outer shear layer (the jet boundary), which is accordance with the measurement by Mitchell et al. (2014) [60]. The acoustic waves that originate around the end of jet potential 

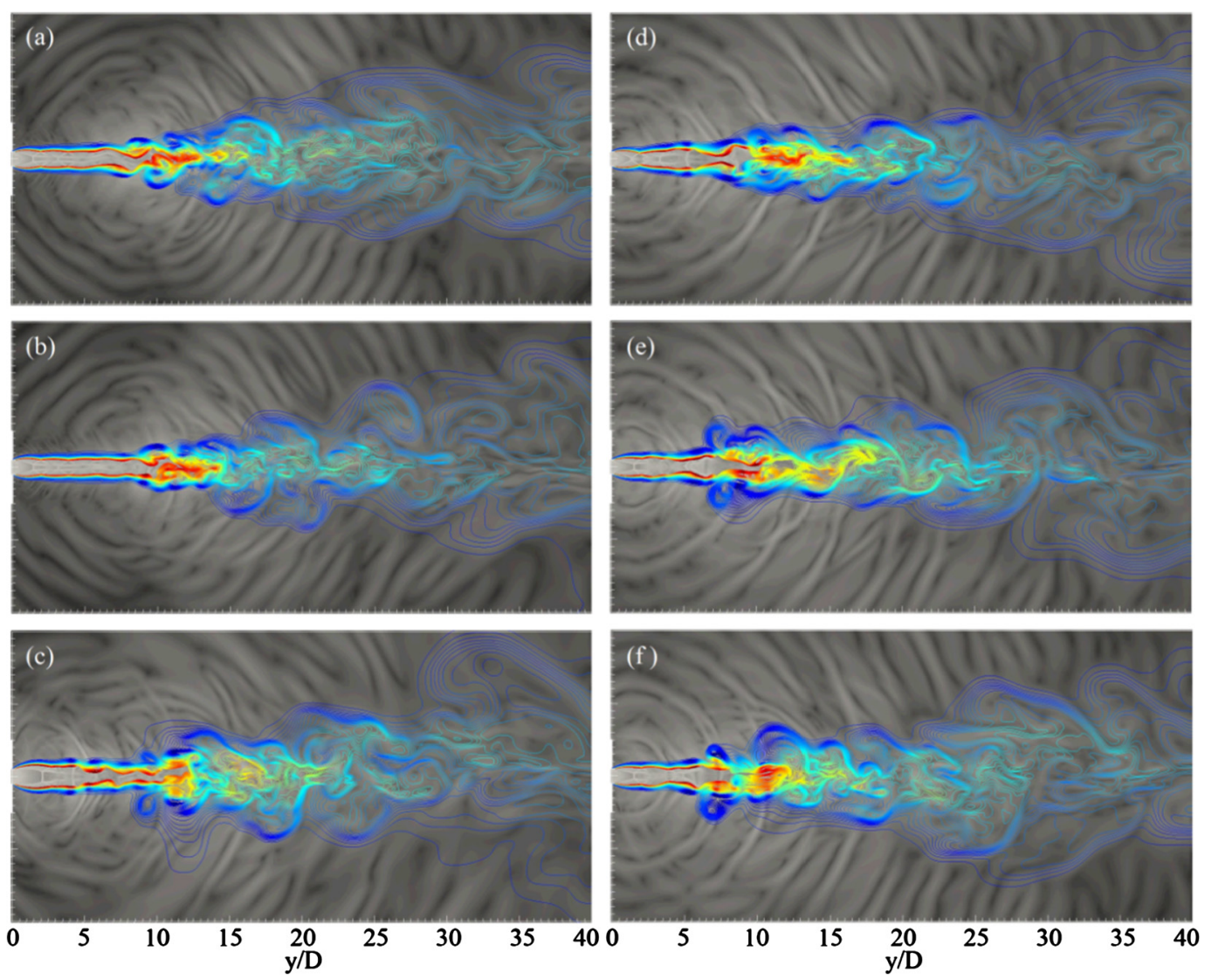

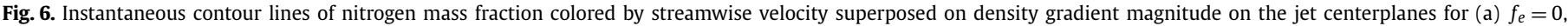
no excitation, (b) $f_{e}=1.0 \mathrm{kHz}$, (c) $f_{e}=14.569 \mathrm{kHz}$, (d) $f_{e}=37.086 \mathrm{kHz}$, (e) $f_{e}=40.0 \mathrm{kHz}$, and (f) $f_{e}=45.695 \mathrm{kHz}$.

core (i.e., at around $y / D=8$ ) propagate both upstream and downstream [48]. More discussions on the underexpanded steady jets and the effect of NPR can be found in the LES study by Vuorinen et al. (2013) [42] and our previous work [35].

Figs. 6(b)-(f) present the flow structures of jets excited at different frequencies. At low excitation frequency of $f_{e}=1.0 \mathrm{kHz}$, the shape and length of jet potential core is similar to the steady jet, and the acoustic source is located at around $y / D=8$ as well. At higher excitation frequencies, the influences of the external excitation on the jet flow and acoustic fields increase significantly. In particular, the nozzle exit becomes the disturbance and consequently acoustic source because of the forced pulsation. Meanwhile, notable differences can be found in the shape and length of the jet potential core between the steady jet and the jets excited by high frequencies, implying the possible changes in the nearfield shock behavior. In addition, the jet boundary does not expand gradually along the streamwise direction from the end of jet potential core as for the steady jet, but experiences a rapid outwards displacement at around $y / D=8$, which will result in a different mixing characteristic between the jet and its surroundings.

Fig. 7 shows the instantaneous contours of density gradient on cross-section planes for different excited jets. As can be seen, the instantaneous density gradient of the excited jet with $f_{e}=1.0 \mathrm{kHz}$ exhibits the same helical distribution as the steady jet, which reveals that the low-frequency excitation of $f_{e}=1.0 \mathrm{kHz}$ doesn't change the dominant instability mode of the underexpanded jet. However, with the increasing of excitation frequency, the density gradient pattern appears axisymmetric, which indicates that the dominant instability mode of those jets have been switched from the helical mode to axisymmetric mode by the pulsed excitation.

\subsection{Shock structures}

Fig. 8(a) presents the time-averaged profiles of nitrogen mass fraction along the centerline of different forcing jets. As can be seen, the length of jet potential core for the forcing jet with $f_{e}=45.695 \mathrm{kHz}$ is around 6D, which is the shortest. Meanwhile, the nitrogen mass fraction at different streamwise positions after the jet potential core is relatively smaller for $f_{e}=45.695 \mathrm{kHz}$ jet, which implies much better mixing. When the jet is excited at the screech frequency of $f_{e}=37.086 \mathrm{kHz}$, the length of jet potential core is slightly shorter than the steady jet. The jet core also shortens to some extent for other high frequencies exciting jets. However, for the low frequency of $f_{e}=1.0 \mathrm{kHz}$, the jet core does not seem to be shortened compared with the steady jet, and the nitrogen mass fraction along the jet centerline approaches to the steady jet value as well.

Fig. 8(b) presents that the mean pressure profiles along the jet centerline. It is evident that pressure of the forcing jets is close to that of the steady jet in the near field region of $y / D<5$ except the $f_{e}=14.569 \mathrm{kHz}$ jet. This finding indicates that the timeaveraged Mach disk height and the location of the second shock of excited jets are similar with the steady jet. After $y / D>5$, the time-averaged length and strength of shock cells in the jets excited by $f_{e}=37.086,40.0$, and $45.695 \mathrm{kHz}$ are both lower than that of the steady jet. In addition, the location where the pressure starts to decrease to ambient pressure is around $y / D=8$ for the $f_{e}=37.086,40.0$, and $45.695 \mathrm{kHz}$ jets, which is earlier than $y / D=11$ for the steady jet. On the other hand, remarkable differences could be found in the pressure profile for the jet excited by the axisymmetric mode of $f_{e}=14.569 \mathrm{kHz}$, which suggests the quite different shock behaviors. After $y / D=15$, the pressure of all jets tends to be uniform. Fig. 8(b) also shows there are five 


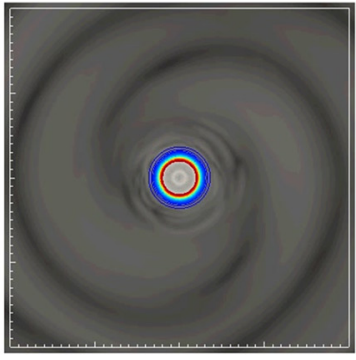

(a) no excitation

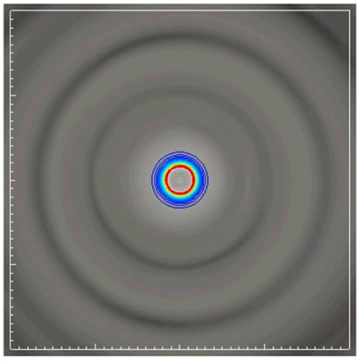

(d) $f_{e}=37.086 \mathrm{kHz}$

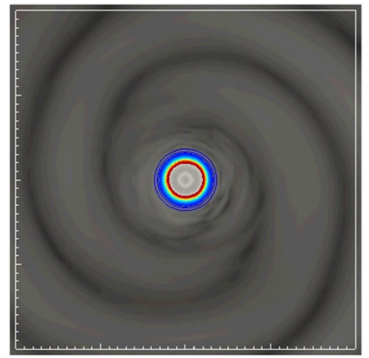

(b) $f_{e}=1.0 \mathrm{kHz}$

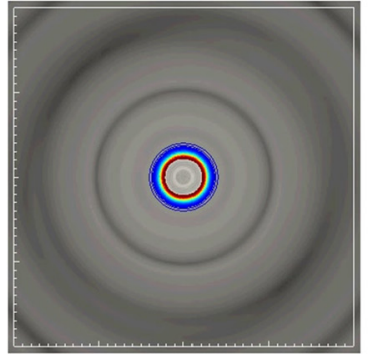

(e) $f_{e}=40.0 \mathrm{kHz}$

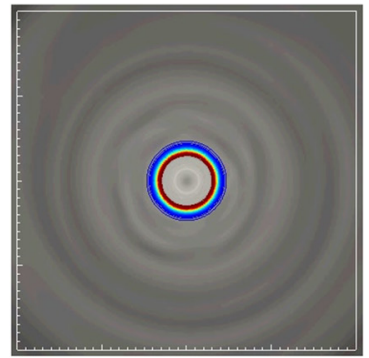

(c) $f_{e}=14.569 \mathrm{kHz}$

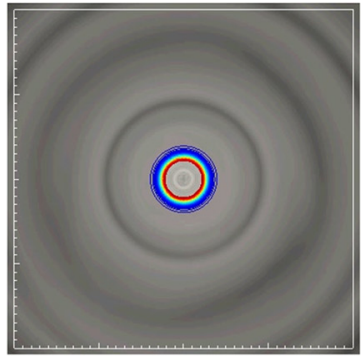

(f) $f_{e}=45.695 \mathrm{kHz}$

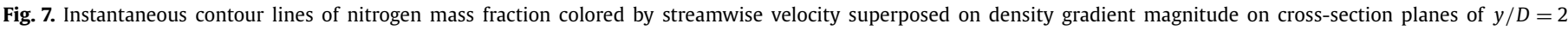
for different jets.
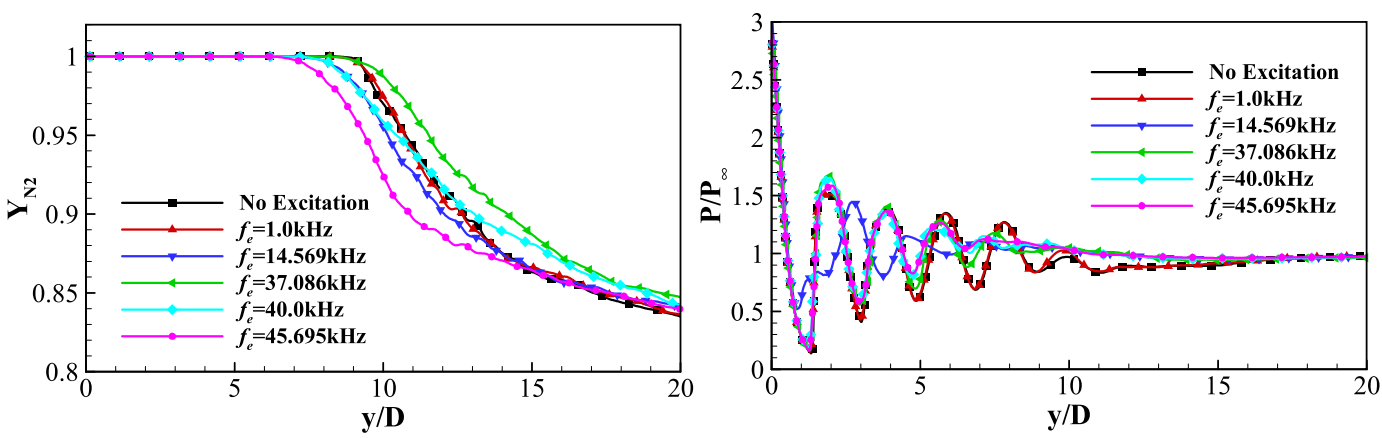

Fig. 8. Time-averaged profiles of flow properties along the jet centerline. (a) Left: nitrogen mass fraction, (b) right: pressure.

shock cells in the steady jet and the low-frequency excited jet $\left(f_{e}=1.0 \mathrm{kHz}\right.$ ). However, four shock cells could be identified in the forcing jets of $f_{e}=37.086,40.0$, and $45.695 \mathrm{kHz}$, while only three shock cells exist in the jet excited at $f_{e}=14.569 \mathrm{kHz}$.

The sinusoidal excitation periodically modulates the inlet mass flow rate and the pressure at the nozzle exit, which subsequently affect the development of shock structures in the near field. The variation in shock structures is confirmed by instantaneous LES results, and Fig. 9 shows unsteady shock motions in a complete excitation cycle as one example for $f_{e}=37.086 \mathrm{kHz}$ jet. As can be seen, the Mach disk, which is the dominant sign of a highly underexpanded jet, does not exist all the time any more, but disappears and forms periodically with time. Interestingly, the variation trend of the third shock cell is contrary to the Mach disk, i.e. the third shock cell widens when the Mach disk narrows, and vice versa. Fig. 9 also indicates that the shocks mainly oscillate along the streamwise direction, while the radial wave motion in underexpanded steady jets revealed by Panda (1998) [59] is not obvious for the current high-frequency excited underexpended jets. In addition, the shape and size of the jet potential core delimited by the green lines in Fig. 9 are found to vary periodically and simultaneously with the unsteady shock motions.

The near-field shocks in other high frequencies excited jets, i.e. $f_{e}=14.569,40.0$, and $45.695 \mathrm{kHz}$, oscillate similarly as that for the $f_{e}=37.086 \mathrm{kHz}$ jet. The instantaneous density gradient for $f_{e}=14.569 \mathrm{kHz}$ shown in Fig. 10 reveals that the jet Mach disk disappears or forms periodically as well over an excitation cycle. However, the shocks oscillate at a much higher amplitude for the $f_{e}=14.569 \mathrm{kHz}$ jet. In particular, the largest Mach barrel in the jet excited by $f_{e}=14.569 \mathrm{kHz}$ shown in Fig. $10(\mathrm{c})-(\mathrm{d})$ is larger than that in the $f_{e}=37.086 \mathrm{kHz}$ jet represented by Fig. 9(c) and (d). Meanwhile, the smallest diamond shock cell in the $f_{e}=14.569 \mathrm{kHz}$ jet shown in Fig. 10(a) and (h) is smaller than that in the $f_{e}=37.086 \mathrm{kHz}$ jet as well. Fig. 11 shows the instantaneous pressure along the jet centerline for $f_{e}=14.569 \mathrm{kHz}$ and $f_{e}=37.086 \mathrm{kHz}$ jets. It is evident that the first shock of the $f_{e}=14.569 \mathrm{kHz}$ jet moves from around $y / D=0.9$ to $y / D=2.1$ during half of the oscillation cycle. On the other hand, the first shock inside the $f_{e}=37.086 \mathrm{kHz}$ jet transports from $y / D=1.35$ to $y / D=1.55$ during half of the oscillation cycle. This finding quantitatively confirms the previous observation based on Fig. 9 and Fig. 10 that shocks inside the $f_{e}=14.569 \mathrm{kHz}$ jet oscillate at a much larger amplitude.

\subsection{Mixing characteristic}

A previous study [34] indicates that the mixing between the jet and its surroundings still takes place at the jet boundary defined by the nitrogen mass fraction of $Y_{N 2}=0.77165$, which corresponds to a mixture fraction level of $X=0.02$. Therefore, the area covered by the $Y_{N 2}=0.77165$ contour line is defined as the mixing area $A_{\text {mix }}$, and is introduced to quantitatively evaluate the jet 

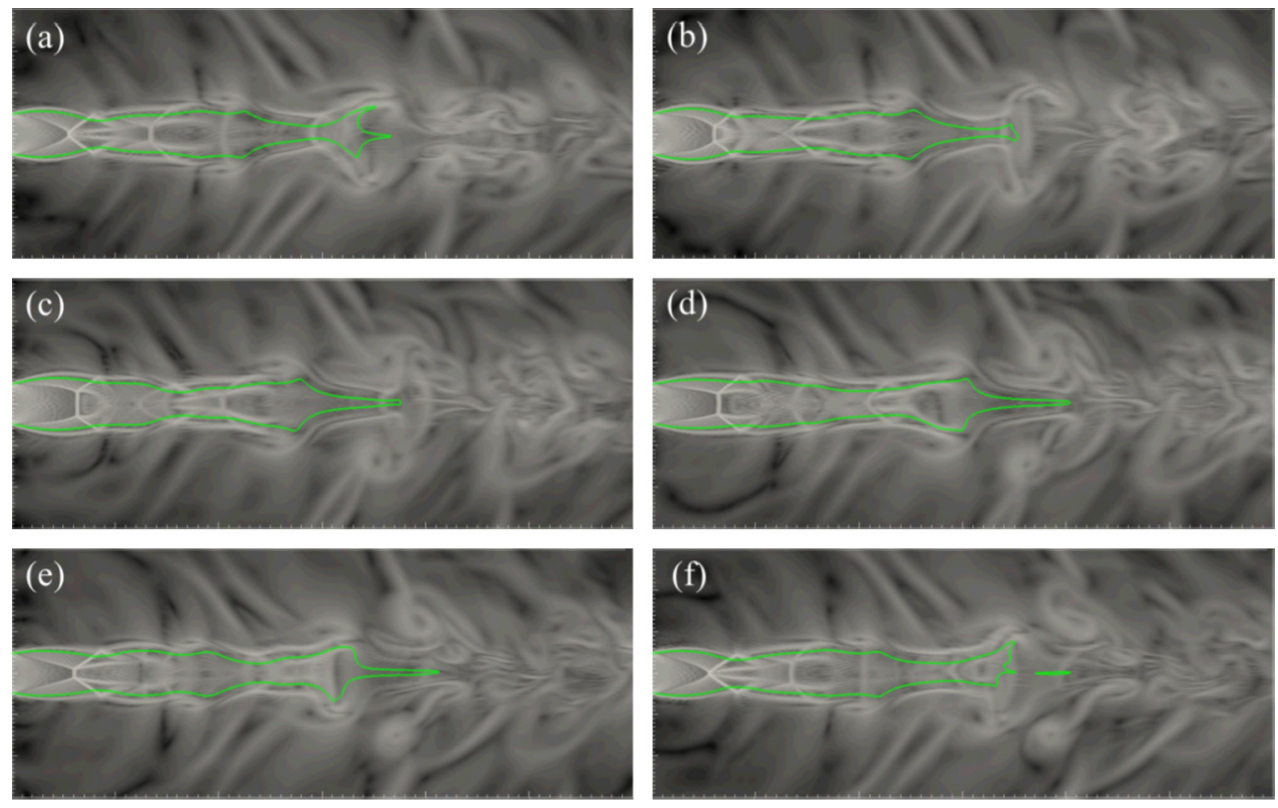

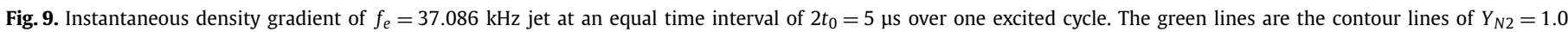
showing the jet core. (For interpretation of the references to color in this figure legend, the reader is referred to the web version of this article.)
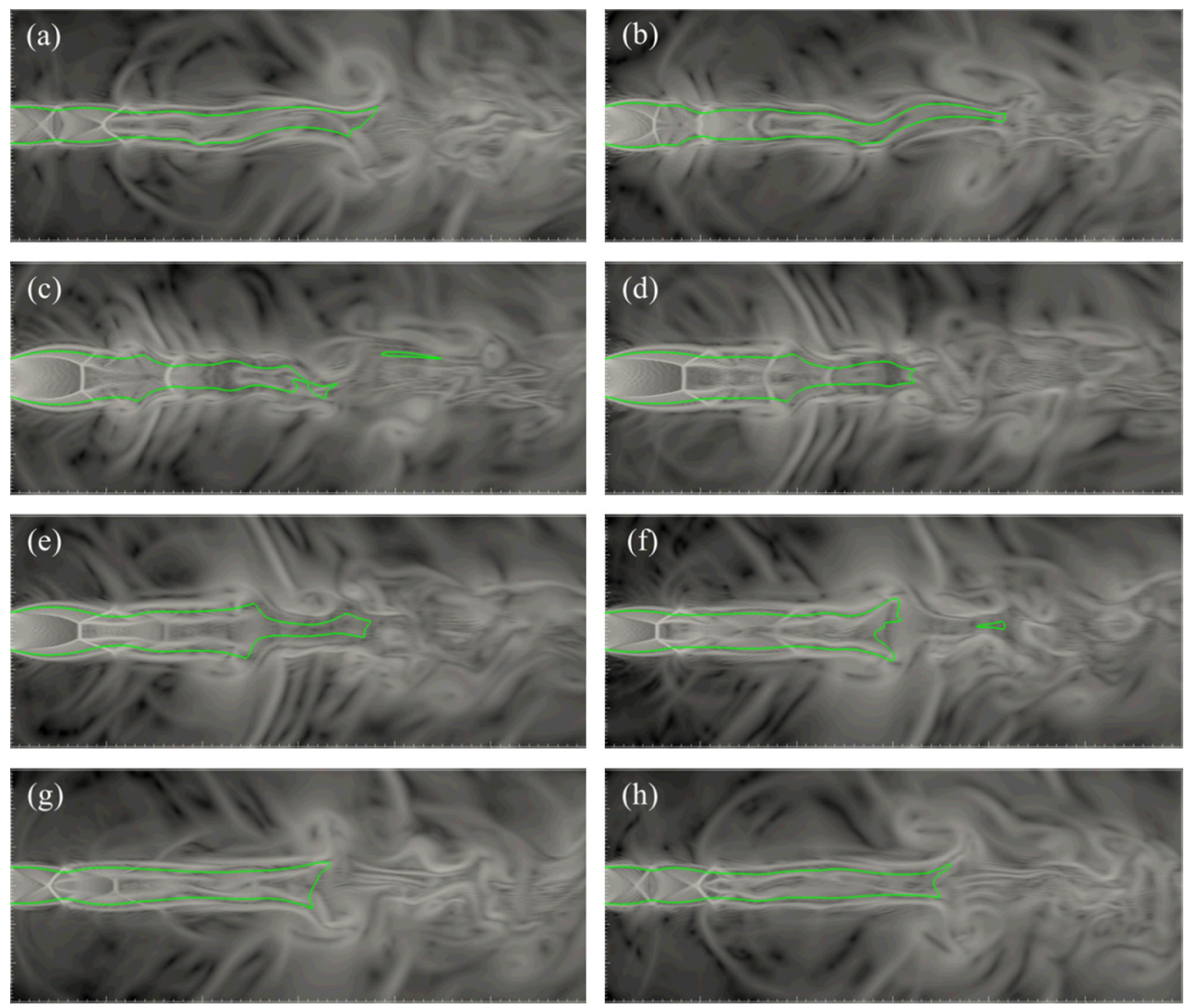

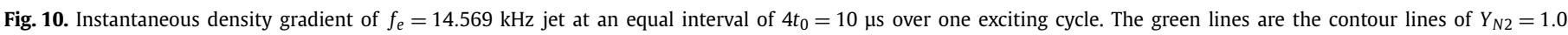
showing the jet core. (For interpretation of the references to color in this figure legend, the reader is referred to the web version of this article.)

mixing performance. The non-dimensional mixing area, i.e. the mixing area normalized by the nozzle exit area $A_{1}$, on different cross-section planes is plotted as a function of time in Fig. 12 for the steady jet and the jets excited by $f_{e}=14.569 \mathrm{kHz}$ and $45.695 \mathrm{kHz}$. As can be seen, the mixing area in the near field of $y / D=4$ is stable relatively for the steady jet, but oscillates in synchronization with the periodic oscillations of the near-field shock structures shown in Fig. 9 and Fig. 10 for the excited jets. In the downstream of $y / D=14$ and 24 , the mixing area of the steady jet and the excited jets all shows somewhat low-frequency fluctuation, which is related with the large scale coherent structures developed along the jet shear layer as shown in Fig. 6. In addition, the instantaneous mixing area for the steady jet and $f_{e}=14.569 \mathrm{kHz}$ jet is similar at $y / D=14$, but is both smaller than the $f_{e}=45.695 \mathrm{kHz}$ 

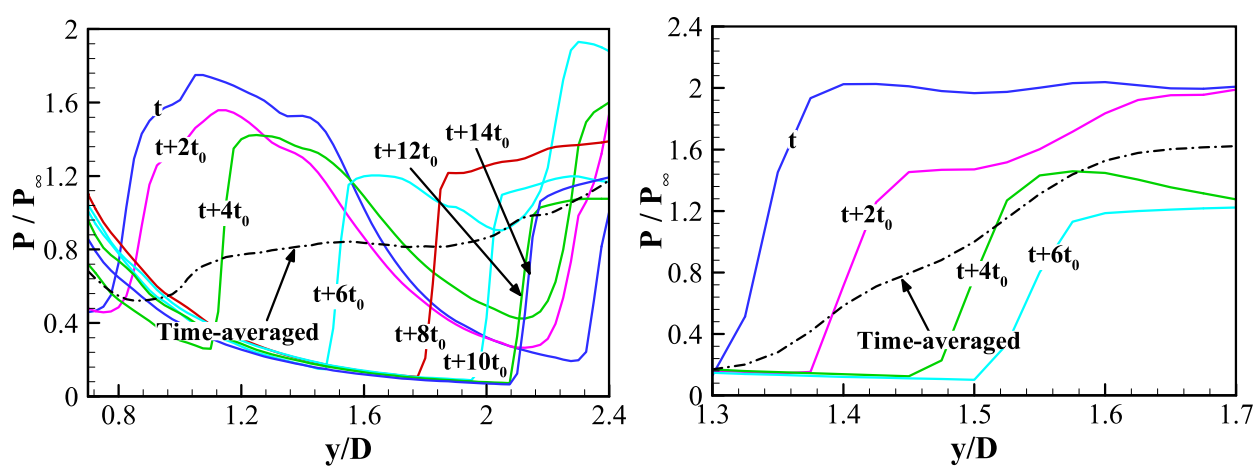

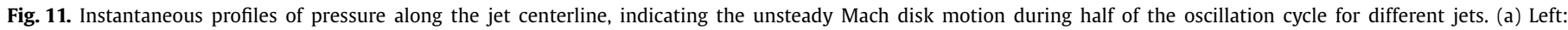
$f_{e}=14.569 \mathrm{kHz}$, (b) right: $f_{e}=37.086 \mathrm{kHz}$.
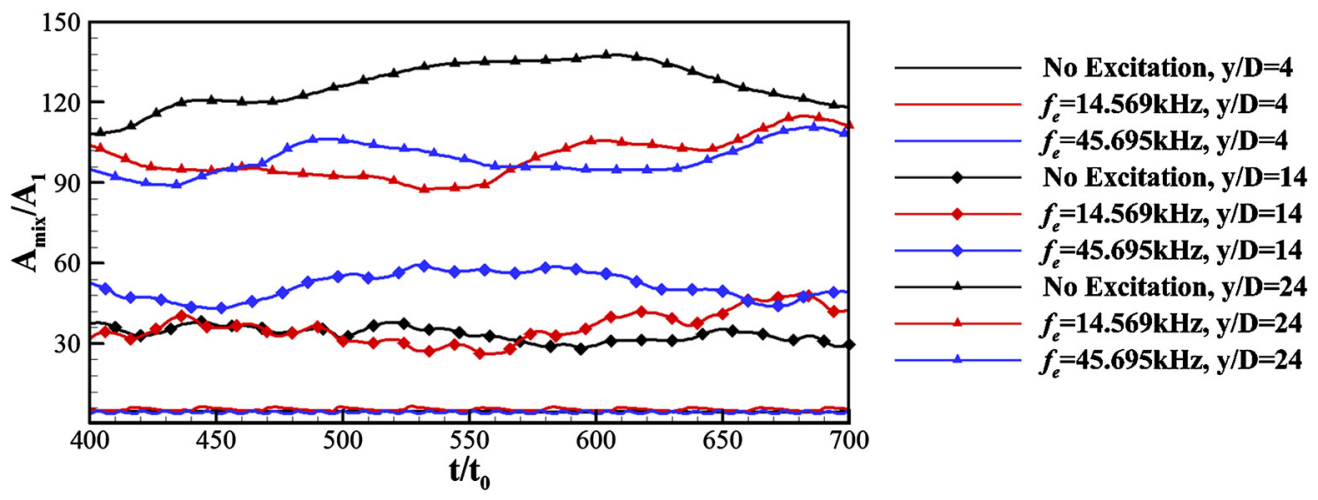

Fig. 12. Time history of normalized mixing area on different cross section planes of the excited jets.

jet. On the other hand, it is found that the mixing area of the two forcing jets is smaller than the steady jet at $y / D=24$ instead.

Fig. 13 presents the time-averaged mixing area of jets on different cross-section planes. As can be seen, the mixing enhancement due to frequencies excitation is unobvious in the near field of $y / D<4$, specially the mean mixing area for the forcing jets excited at $f_{e}=37.086 \mathrm{kHz}, 40.0 \mathrm{kHz}$, and $45.695 \mathrm{kHz}$ is even slightly smaller than the steady jet. The mixing area of the steady jet is larger than high-frequency excited jets at $y / D=6$ because of the appearance of the undulating vortex ring [35]. This undulating vortex ring sustains longer for the steady jet and the jet excited by the low frequency of $f_{e}=1.0 \mathrm{kHz}$, but vanishes quickly in the highfrequency excited jets. As a result, the mixing area of the steady jet at $y / D=6$ is even larger than that at $y / D=8$ and 10 .

Fig. 13 shows that the mixing between the jet and its surroundings is highly enhanced from $y / D=8$ to 12 by the external excitation at $f_{e}=14.569 \mathrm{kHz}, 40.0 \mathrm{kHz}$, and $45.695 \mathrm{kHz}$. Generally, the mixing area of those excited jets is above twice of the steady jet. In particular, the mixing performance of jet excited by the axisymmetric mode of $f_{e}=14.569 \mathrm{kHz}$ is prominent, with the mixing area being seven times that of the steady jet at $y / D=10$. This can be explained by the fact that the sinusoidal disturbance imposed to the nozzle entrance pressure in the present LES is axisymmetric and resonates strongly with the inherent axisymmetric mode of the steady jet at the same frequency of $f_{e}=14.569 \mathrm{kHz}$. The intense resonances by the external excitation at $f_{e}=14.569 \mathrm{kHz}$ remarkably change the jet structure as seen in Fig. 6 and Fig. 10, and consequently result in a larger mixing area. When forcing the jet at the shock screech frequency of $f_{e}=37.086 \mathrm{kHz}$, the mixing area between $y / D=8$ and 12 is also increased compared with the steady jet, but is smaller than that of the other high-frequency excited jets, i.e. $f_{e}=14.569 \mathrm{kHz}, 40.0 \mathrm{kHz}$, and $45.695 \mathrm{kHz}$ jets. For example, the non-dimensional mixing area of the $f_{e}=37.086 \mathrm{kHz}$ jet is about 31.0 at $y / D=10$, which is larger than 10 for the steady jet but smaller than 35.5 for the $f_{e}=40.0 \mathrm{kHz}$ jet.

Further downstream at $y / D=14$ and 16 , the mixing area is larger than the steady jet for the $f_{e}=45.695 \mathrm{kHz}$ jet but smaller than the steady jet for the other frequencies excited jets, which implies that the second screech frequency has a much broader range on jet mixing enhancing. After $y / D=16$, it is interesting to find that the high-frequency excitation suppresses the mixing reversely. For example, the mixing area of the excited jets with $f_{e}=14.569 \mathrm{kHz}, 35.695 \mathrm{kHz}, 40.0 \mathrm{kHz}$, and $45.695 \mathrm{kHz}$ is generally smaller than that of the steady jet from $y / D=18$ to 30 .

Fig. 13 also indicates that the external excitation at the low frequency of $f_{e}=1.0 \mathrm{kHz}$ has trivial effect on the mixing enhancing in the near field, as the mixing area of the $f_{e}=1.0 \mathrm{kHz}$ jet is generally close to or smaller than the steady jet for $y / D \leq 22$. However, contrary to the fact that high frequencies decrease the jet mixing area downstream of $y / D=18$, the mixing area of the $f_{e}=1.0 \mathrm{kHz}$ jet exceeds that of the steady jet from $y / D=24$ to far downstream. This finding agrees reasonably with the experimental observation by Narayanan et al. (2003) [22] on subsonic jets issued into a subsonic cross flow, i.e. exciting a jet at high frequencies improves the jet spread in the near field, while the low-frequency excitation enhances the mixing in the far field.

\subsection{Spectrum analysis}

Fast Fourier Transformations (FFT) of pressure fluctuation probed on either side of the jets are implemented. Fig. 14 shows the spectrum and relative phase of pressure fluctuations near the jet shear layer for different jets. As can be seen, different from the steady jet that have two discrete dominant frequencies of $f_{s}=37.086 \mathrm{kHz}$ and $f_{2 s}=45.695 \mathrm{kHz}$, the jet excited at $f_{e}=1.0 \mathrm{kHz}$ only has one peak frequency of $f=37.003 \mathrm{kHz}$, which is slightly smaller than the shock screech frequency of the steady jet. The peak frequency 
$\square$ f00 $\square$ f01 $\square$ fl4 $\square \mathrm{f} 37 \square \mathrm{f} 40 \quad \square \mathrm{f} 45$
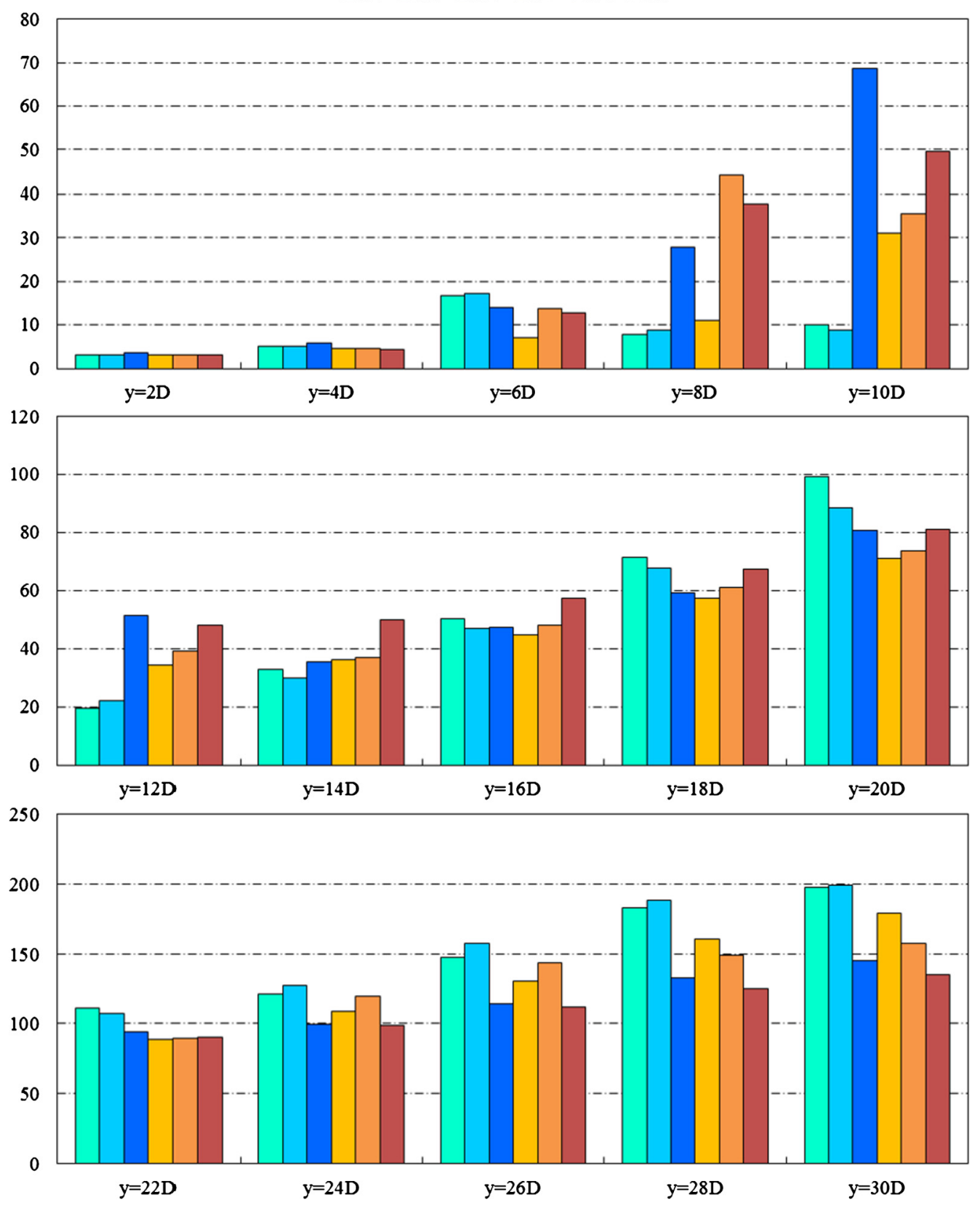

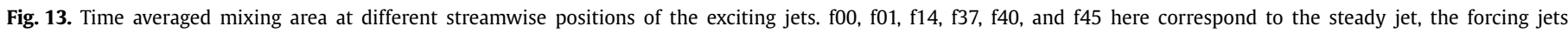
excied by $f_{e}=1.0 \mathrm{kHz}, 14.569 \mathrm{kHz}, 37.086 \mathrm{kHz}, 40.0 \mathrm{kHz}$, and $45.695 \mathrm{kHz}$ successively.

of $f=37.003 \mathrm{kHz}$ has a phase angle close to $-180^{\circ}$, suggesting that the dominant mode of the $f_{e}=1.0 \mathrm{kHz}$ jet is the same helical mode as the steady jet. This is in accordance with the previous observation based on Fig. 7. In addition, two axisymmetric modes of $4.869 \mathrm{kHz}$ and $14.607 \mathrm{kHz}$ exist in the spectrum of the $f_{e}=1.0 \mathrm{kHz}$ jet, which are close to the $5.298 \mathrm{kHz}$ and $14.569 \mathrm{kHz}$ for the steady jet, respectively.

When excited by the inherent characteristic frequencies of the steady jet, i.e. $f_{e}=14.569 \mathrm{kHz}, 37.086 \mathrm{kHz}$, and $45.695 \mathrm{kHz}$, the dominant frequency of the forcing jets turns into the corresponding exciting frequency, and the dominant mode of the jets all switches to axisymmetric mode from the helical mode. For example, the $f_{e}=14.569 \mathrm{kHz}$ jet is dominated by the same exciting frequency of $14.569 \mathrm{kHz}$, and the dominant mode is axisymmetric with a phase angle close to $0^{\circ}$, which agrees well with the finding based on Fig. 7. Similarly, the peak frequency of the jet be- comes the axisymmetric mode of $37.086 \mathrm{kHz}$ as well when the jet is excited by the shock screech frequency of $f_{s}=37.086 \mathrm{kHz}$. On the other hand, it is interesting to find that the peak frequency is $f=39.735 \mathrm{kHz}$ when the jet is excited at $f_{e}=40.0 \mathrm{kHz}$, which is slightly smaller than the forcing frequency applied. However, the phase angle of the dominant frequency is still close to $0^{\circ}$ for the $f_{e}=40.0 \mathrm{kHz}$ jet, implying that the excited jet is also dominated by the axisymmetric mode.

From Fig. 14, there are some high-order harmonics of the dominant frequency in the spectrum of jets excited by high frequencies, i.e. $f_{e}=14.569 \mathrm{kHz}, f_{e}=37.086 \mathrm{kHz}, 40.0 \mathrm{kHz}$, and $45.695 \mathrm{kHz}$. For example, besides the dominant frequency of $f_{s}=14.569 \mathrm{kHz}$, the first harmonics ( $f=29.139 \mathrm{kHz} \approx 2 f_{s}$ ) and the second harmonics $\left(f=43.708 \mathrm{kHz} \approx 3 f_{s}\right.$ ) also exist in the spectrum of the $f_{e}=14.569 \mathrm{kHz}$ jet. The first harmonics $\left(f=91.390 \mathrm{kHz} \approx 2 f_{s}\right.$ ) can also be identified when the jet is excited at the second shock 


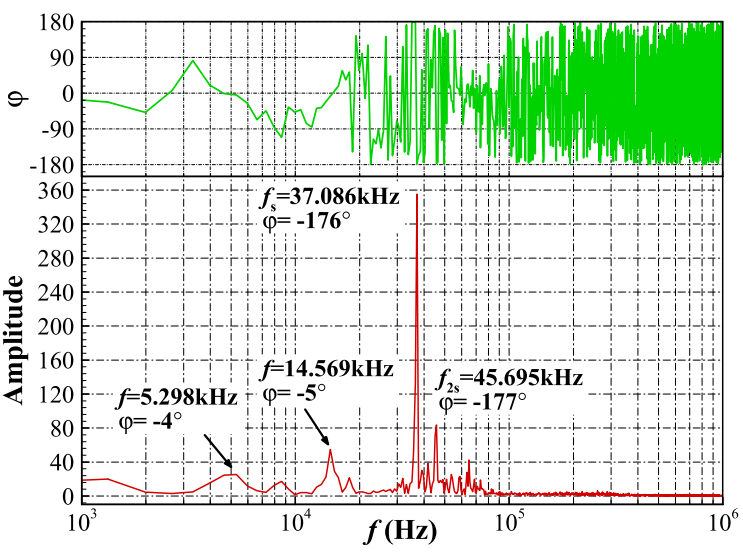

(a) no excitation

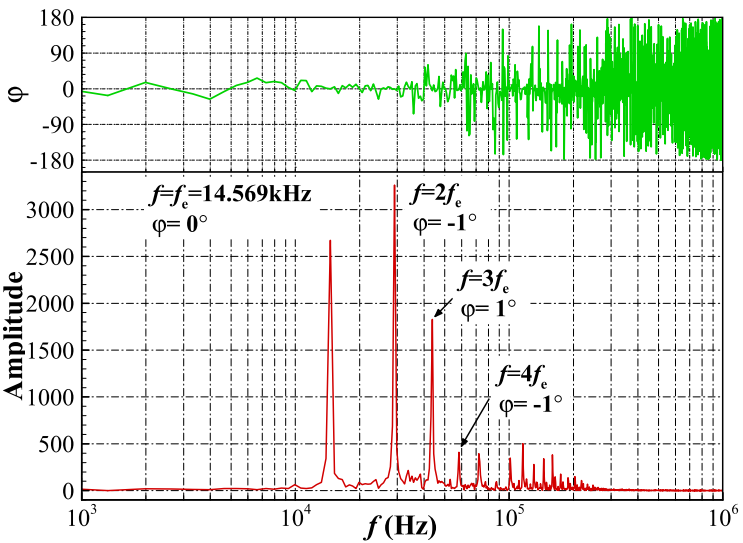

(c) $f_{e}=14.569 \mathrm{kHz}$

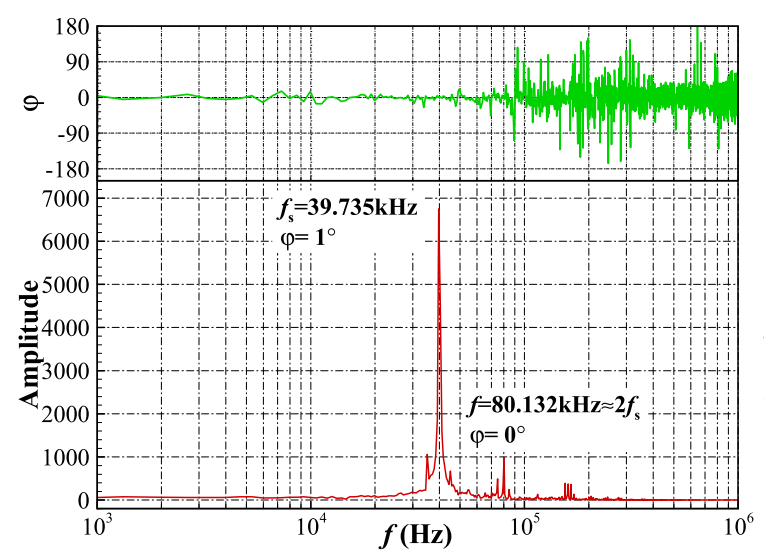

(e) $f_{e}=40.0 \mathrm{kHz}$

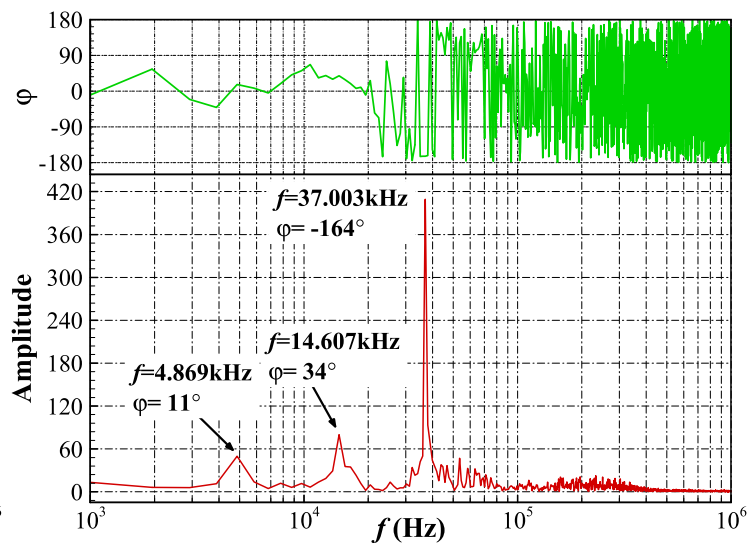

(b) $f_{e}=1.0 \mathrm{kHz}$

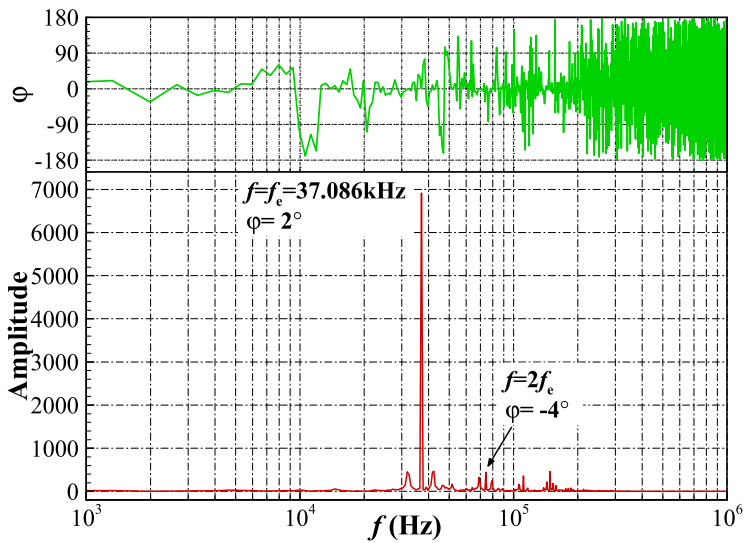

(d) $f_{e}=37.086 \mathrm{kHz}$

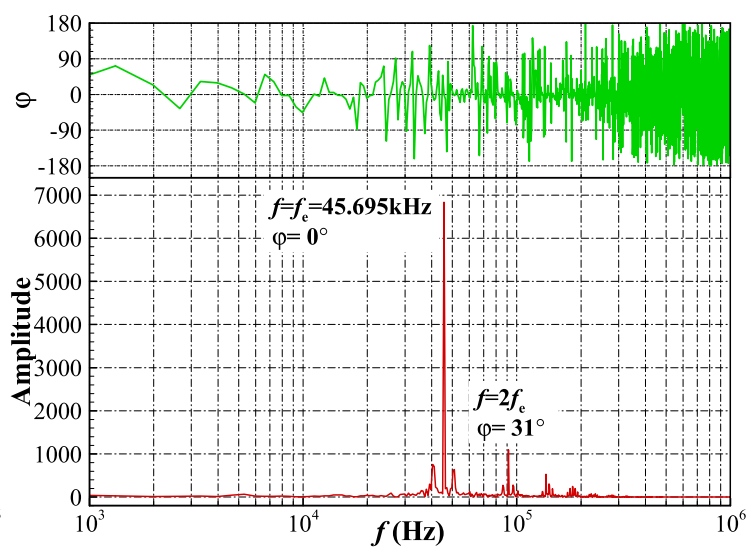

(f) $f_{e}=45.695 \mathrm{kHz}$

Fig. 14. The cross spectrum and relative phase of pressure fluctuation on either side of the jets at $(x / D=1, y / D=2, z / D=0)$ and $(x / D=-1, y / D=2, z / D=0)$.

screech frequency of $f_{e}=45.695 \mathrm{kHz}$. Fig. 14 also shows that there are more harmonics in the $f_{e}=14.569 \mathrm{kHz}$ jet, e.g. the $2 f_{e}$, $3 f_{e}$ and $4 f_{e}$ identified. The intense resonance between the inherent axisymmetric mode of $f=14.569 \mathrm{kHz}$ in the steady jet and the axisymmetric excitation at the same frequency may be the main contributing factors to produce more harmonics.

\section{Conclusions}

In this study, the effect of external excitation at characteristic frequencies on highly underexpanded jet flow is investigated by LES. A sinusoidal disturbance is imposed to the static pressure at the nozzle entrance to excite the jets. The inherent peak frequencies in the steady jet of $14.569 \mathrm{kHz}, 37.086 \mathrm{kHz}$, and $45.695 \mathrm{kHz}$ as well as two reference frequencies of $1.0 \mathrm{kHz}$ and $40.0 \mathrm{kHz}$ are selected as the forcing frequencies. A high-pressure gas jet injection model with a practical nozzle and a block-structured mesh of high resolution are applied to model the realistic jet behaviors. The density-based solver for supersonic compressible flows, astroFoam, is used to carry out the simulations. Various flow properties of the steady jet are compared with the available literature data to validate the fidelity of the LES results, and the reasonable agreement is observed. The main conclusions of this study are summarized as follows. 
(1) The external excitation has a remarkable influence on the jet core and the near field shock structures. When the jet is excited at the low frequency of $f_{e}=1.0 \mathrm{kHz}$, the size of jet core, the location of the acoustic source, and the quasi-periodic shock structures in the near field of jet are similar with the steady jet. When excited by other high frequencies, the acoustic source moves to the nozzle exit, the jet core and the nearfield shocks oscillate periodically at the forcing frequency. In particular, the $f_{e}=45.695 \mathrm{kHz}$ jet has the shortest average jet core, while the $f_{e}=14.569 \mathrm{kHz}$ jet has the fewest shock cells and takes the largest amplitude in shock oscillation.

(2) The mixing area of the forcing jets periodically oscillates at the excited frequency in the near field, but shows some low frequency fluctuation as the steady jet in the downstream. The excitations by $f_{e}=14.569 \mathrm{kHz}, 40.0 \mathrm{kHz}$, and $45.695 \mathrm{kHz}$ improve the average mixing area at $8 \leq y / D \leq 12$ significantly, especially for $f_{e}=14.569 \mathrm{kHz}$. In the downstream of $y / D \geq 18$, the external excitations at high frequencies decrease the mixing area on the contrary. However, the mixing area of the $f_{e}=1.0 \mathrm{kHz}$ jet exceeds that of the steady jet since $y / D=24$, till the far downstream.

(3) The $f_{e}=1.0 \mathrm{kHz}$ jet is dominated by a similar helical mode of $f=37.003 \mathrm{kHz}$, which is slightly smaller than the shock screech tone of $f_{s}=37.086 \mathrm{kHz}$ in the steady jet. When excited by the inherent frequencies of $f_{e}=14.569 \mathrm{kHz}$, $37.086 \mathrm{kHz}$, and $45.695 \mathrm{kHz}$ in the steady jet, the dominant frequency of the excited jets turns into the exciting frequency applied, and the dominant mode all switches to axisymmetric mode. However, the peak frequency is $f=39.735 \mathrm{kHz}$ when the jet is excited at $f_{e}=40.0 \mathrm{kHz}$, which is slightly smaller than the excitation frequency. In addition, high-order harmonics of the dominant frequency exist in the spectrum of jets excited by high frequencies, and the $f_{e}=14.569 \mathrm{kHz}$ jet has most harmonics.

\section{Conflict of interest statement}

The authors declare that there is no any conflict of interest.

\section{Acknowledgements}

This project was funded by the National Natural Science Foundation of China (Grant No. 11502270) and China Postdoctoral Science Foundation (No. 2016M591258).

\section{References}

[1] M. Berglund, E. Fedina, C. Fureby, J. Tegnér, Finite rate chemistry large-eddy simulation of self-ignition in supersonic combustion ramjet, AIAA J. 48 (3) (2010) 540-550.

[2] Z.P. Wang, F. Li, H.B. Gu, X.L. Yu, X.Y. Zhang, Experimental study on the effect of combustor configuration on the performance of dual-mode combustor, Aerosp. Sci. Technol. 42 (2015) 169-175.

[3] W. Huang, J. Liu, L. Jin, et al., Molecular weight and injector configuration effects on the transverse injection flow field properties in supersonic flows, Aerosp. Sci. Technol. 32 (1) (2014) 94-102.

[4] X. Liu, A. Godbole, C. Lu, G. Michal, P. Venton, Source strength and dispersion of $\mathrm{CO}_{2}$ releases from high-pressure pipelines: CFD model using real gas equation of state, Appl. Energy 126 (1) (2014) 56-68.

[5] X. Guo, X. Yan, J. Yu, Y. Zhang, et al., Under-expanded jets and dispersion in supercritical $\mathrm{CO}_{2}$ releases from a large-scale pipeline, Appl. Energy 183 (1) (2016) 1279-1291.

[6] J.A. Moríñigo, J.J. Salvá, Numerical study of the start-up process in an optimized rocket nozzle, Aerosp. Sci. Technol. 12 (6) (2008) 485-489.

[7] A. Hadjadj, Y. Perrot, S. Verma, Numerical study of shock/boundary layer interaction in supersonic overexpanded nozzles, Aerosp. Sci. Technol. 42 (2015) $158-168$.

[8] John M. Seiner, S.M. Dash, D.C. Kenzakowski, Historical survey on enhanced mixing in scramjet engines, J. Propuls. Power 17 (6) (2001) 1273-1286.
[9] W. Huang, L. Yan, Progress in research on mixing techniques for transverse injection flow fields in supersonic crossflows, J. Zhejiang Univ. Sci. A 14 (8) (2014) 554-564.

[10] W. Huang, Transverse jet in supersonic crossflows, Aerosp. Sci. Technol. 50 (2016) 183-195.

[11] W. Huang, W.D. Liu, S.B. Li, et al., Influences of the turbulence model and the slot width on the transverse slot injection flow field in supersonic flows, Acta Astronaut. 73 (2) (2012) 1-9.

[12] W. Huang, Z.G. Wang, J.P. Wu, et al., Numerical prediction on the interaction between the incident shock wave and the transverse slot injection in supersonic flows, Aerosp. Sci. Technol. 28 (1) (2013) 91-99.

[13] G.B. Northam, I. Greenberg, C.S. Byington, Evaluation of parallel injector configurations for supersonic combustion, AIAA paper 1989-2525.

[14] E.J. Gutmark, K.C. Schadow, K.H. Yu, Mixing enhancement in supersonic free shear flows, Annu. Rev. Fluid Mech. 27 (1995) 375-417.

[15] M.R. Gruber, R.A. Baurle, T. Mathur, et al., Fundamental studies of cavitybased flameholder concepts for supersonic combustors, J. Propuls. Power 17 (1) (2001) 146-153.

[16] T.O. Mohieldin, S.N. Tiwari, Effects of tandem injection on compressible turbulent shear layer, AIAA paper 1998-1638.

[17] F. Génin, S. Menon, Simulation of turbulent mixing behind a strut injector in supersonic flow, AIAA J. 48 (3) (2010) 526-539.

[18] M. Gruber, J. Donbar, T. Jackson, et al. Performance of an aerodynamic ramp fuel injector in a scramjet combustor, AIAA paper, 2000-3708.

[19] W. Huang, S.B. Li, L. Yan, et al., Performance evaluation and parametric analysis on cantilevered ramp injector in supersonic flows, Acta Astronaut. 84 (3) (2013) 141-152.

[20] R.C. Rogers, D.P. Capriotti, R.W. Guy, Experimental supersonic combustion research at NASA Langley, AIAA paper 1998-2506.

[21] Adnan Eroglu, Robert E. Breidenthal, Structure, penetration, and mixing of pulsed jets in crossflow, AIAA J. 39 (3) (2001) 417-423.

[22] S. Narayanan, P. Barooah, J.M. Cohen, Dynamics and control of an isolated jet in crossflow, AIAA J. 41 (12) (2003) 2316-2330.

[23] Hamid Johari, Scaling of fully pulsed jets in crossflow, AIAA J. 44 (11) (2006) 2719-2725.

[24] J. Davitian, C. Hendrickson, D. Getsinger, et al., Strategic control of transverse jet shear layer instabilities, AIAA J. 48 (9) (2010) 2145-2156.

[25] A.R. Karagozian, Transverse jets and their control, Prog. Energy Combust. Sci. 36 (5) (2010) 531-553.

[26] S. Murugappan, E. Gutmark, C. Carter, Control of penetration and mixing of an excited supersonic jet into a supersonic cross stream, Phys. Fluids 17 (2005) 106101.

[27] T. Kouchi, K. Sasaya, J. Watanabe, et al. Penetration characteristics of pulsed injection into supersonic crossflow, AIAA paper 2010-6645.

[28] Ganesh Raman, Edward J. Rice, Axisymmetric jet forced by fundamental and subharmonic tones, AIAA J. 29 (7) (1989) 1114-1122.

[29] M. Samimy, J.-H. Kim, J. Kastner, et al., Active control of high-speed and highReynolds-number jets using plasma actuators, J. Fluid Mech. 578 (1) (2007) 305-330.

[30] M. Samimy, J.-H. Kim, M. Kearney-Fischer, et al., Acoustic and flow fields of an excited high Reynolds number axisymmetric supersonic jet, J. Fluid Mech. 656 (2010) 507-529.

[31] M.W. Chase, JANAF thermochemical tables, J. Phys. Chem. Ref. Data 3 (2) (1974) $311-480$.

[32] V. Chakravarthy, S. Menon, Large eddy simulations of turbulent premixed flames in the flamelet regime, Combust. Sci. Technol. 162 (2001) 175-222.

[33] C.J. Greenshields, H.G. Weller, L. Gasparini, et al., Implementation of semidiscrete, non-staggered central schemes in a colocated, polyhedral, finite volume framework, for high-speed viscous flows, Int. J. Numer. Methods Fluids 63 (1) (2010) 1-21.

[34] Xiaopeng Li, Kun Wu, Wei Yao, Xuejun Fan, A comparative study of highly underexpanded nitrogen and hydrogen jets using large eddy simulation, Int. J. Hydrog. Energy 41 (9) (2016) 5151-5161.

[35] Xiaopeng Li, Wei Yao, Xuejun Fan, Large-eddy simulation of time evolution and instability of highly underexpanded sonic jets, AIAA J. 54 (10) (2016) 3191-3211.

[36] A. Kurganov, E. Tadmor, New high-resolution central schemes for nonlinear conservation laws and convection-diffusion equations, J. Comput. Phys. 160 (1) (2000) 241-282.

[37] H. Jasak, H.G. Weller, A.D. Gosman, High resolution NVD differencing scheme for arbitrarily unstructured meshes, Int. J. Numer. Methods Fluids 31 (2) (1999) 431-449.

[38] M.H. Baba-Ahmadi, G. Tabor, Inlet conditions for LES using mapping and feedback control, Comput. Fluids 38 (6) (2009) 1299-1311.

[39] J. Liu, R. Ramamurti, D. Munday, E. Gutmark, K. Kailasanath, R. Lohner, Largeeddy simulations of a supersonic jet and its near-field acoustic properties, AIAA J. 47 (8) (2009) 1849-1865.

[40] A. Dauptain, B. Cuenot, Y.M. Gicquel, Large-eddy simulation of a stable supersonic jet impinging on flat plate, AIAA J. 48 (10) (2010) 2325-2337. 
[41] A. Hamzehloo, P.G. Aleiferis, Gas dynamics and flow characteristics of highly turbulent under-expanded hydrogen and methane jets under various nozzle pressure ratios and ambient pressures, Int. J. Hydrog. Energy 41 (15) (2016) 6544-6566.

[42] V. Vuorinen, J. Yu, S. Tirunagari, et al., Large-eddy simulation of highly underexpanded transient gas jets, Phys. Fluids 25 (1) (2013) 016101.

[43] A.D. Cutler, G.C. Harding, G.S. Diskin, High frequency supersonic pulsed injection, AIAA paper 2001-517.

[44] H. Randolph, L. Chew, H. Johari, Pulsed jets in crossflow, J. Propuls. Power 10 (5) (1994) 746-748

[45] A. Powell, On the mechanism of choked jet noise, Proc. Phys. Soc. B 66 (12) (1953) 1039

[46] E. Gutmark, K.C. Schadow, C.J. Bicker, Mode switching in supersonic circular jets, Phys. Fluids 1 (5) (1989) 868-873.

[47] A. Powell, Y. Umeda, R. Ishii, Observations of the oscillation modes of choked circular jets, J. Acoust. Soc. Am. 92 (5) (1992) 2823-2836.

[48] C.K.W. Tam, Supersonic jet noise, Annu. Rev. Fluid Mech. 27 (1995) 17-43.

[49] S. Crist, D.R. Glass, P.M. Sherman, Study of the highly underexpanded sonic jet, AIAA J. 4 (1) (1966) 68-71.

[50] C.D. Donaldson, R.S. Snedeker, A study of free jet impingement. Part 1. Mean properties of free and impinging jets, J. Fluid Mech. 45 (2) (1971) 281-319.

[51] J. Panda, R.G. Seasholtz, Measurement of shock structures and shock-vortex interaction in underexpanded jets using Rayleigh scattering, Phys. Fluids 11 (12) (1999) 3761-3777.
[52] A. Hamzehloo, P.G. Aleiferis, Numerical modelling of transient underexpanded jets under different ambient thermodynamic conditions with adaptive mesh refinement, Int. J. Heat Fluid Flow (2017), http://dx.doi.org/10.1016/ j.ijheatfluidflow.2016.07.015, in press.

[53] E. Franquet, V. Perrier, S. Gibout, et al., Free underexpanded jets in a quiescent medium: a review, Prog. Aerosp. Sci. 77 (2015) 25-53.

[54] P. Ouellette, Direct Injection of Natural Gas for Diesel Engine Fueling, PhD thesis, University of British Columbia, 1996.

[55] P. Ouellette, P.G. Hill, Turbulent transient gas injections, J. Fluids Eng. 122 (2000) 743-753.

[56] E. Ferdman, M.V. Otugen, S. Kim, Effect of initial velocity profile on the devel opment of round jets, J. Propuls. Power 16 (4) (2000) 676-686.

[57] Yüceil K. Bülent, Ötügen M. Volkan, Scaling parameters for underexpanded supersonic jets, Phys. Fluids 14 (12) (2002) 4206-4215

[58] R.C. Deo, J. Mi, G.J. Nathan, The influence of Reynolds number on a plane jet, Phys. Fluids 20 (7) (2008) 075108.

[59] J. Panda, Shock oscillation in underexpanded screeching jets, J. Fluid Mech. 363 (1998) 173-198.

[60] D. Edgington-Mitchell, D.R. Honnery, J. Soria, The underexpanded jet Mach disk and its associated shear layer, Phys. Fluids 26 (2014) 096101. 